JUSTUS-LIEBIG-

5 UNIVERSITÄT

GIESSEN

\author{
Barbara E. Weißenberger
}

\title{
Controlling als Teilgebiet der Betriebswirtschaftslehre: Konzeptionelle Einordnung und Konsequenzen für Forschung und Lehre
}

\author{
Working Paper 1 / 2002
}

- Arbeitspapiere Industrielles Management und Controlling -

Herausgeber:

\author{
Professur für Betriebswirtschaftslehre mit dem Schwerpunkt Industrielles \\ Management und Controlling (Prof. Dr. Barbara E. Weißenberger) \\ Justus-Liebig-Universität, Gießen \\ http://wiwi.uni-giessen.de/controlling/
}

JEL-Classification:

M41

Statement für den Controller-Kongress 21./22. März 2002, Vallendar

Erschienen in:

Weber, Jürgen/Hirsch, Bernhard (Hrsg.): Controlling als akademische Disziplin. Eine Bestandsaufnahme, Wiesbaden: Gabler, 2002, S. 389408. 


\section{Controlling als Teilgebiet der Betriebswirtschafts- lehre: Konzeptionelle Einordnung und Konse- quenzen für Forschung und Lehre}

\section{Gliederung}

1 Zur Entwicklung des Controlling als Fachgebiet innerhalb der Betriebswirtschaftslehre

1.1 Controlling als Phänomen in der unternehmerischen Praxis ....................... 1

1.2 Controlling in der betriebswirtschaftlich-theoretischen Diskussion .............. 3

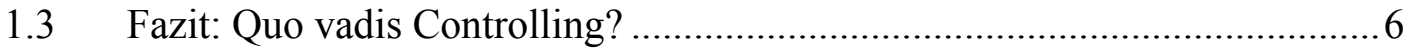

2 Erkenntnisobjekt der Controllingforschung oder: Gibt es eine eigenständige Controllingtheorie?

2.1 Controlling als Reaktion auf den inhaltlichen Stillstand in der Kostenrechnungstheorie....

2.2 Controlling als Lehre von der Messung güterwirtschaftlicher

Sachverhalte für betriebliche Entscheidungen

3 Konsequenzen des vorgeschlagenen Controllingverständnisses für

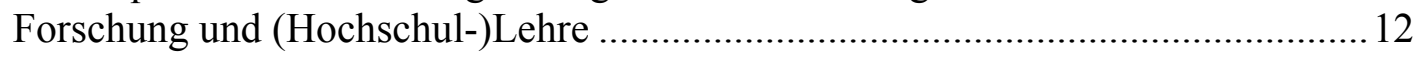

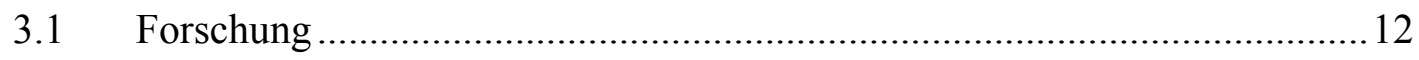

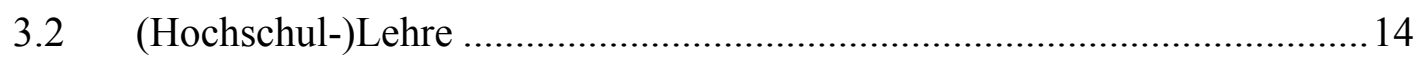

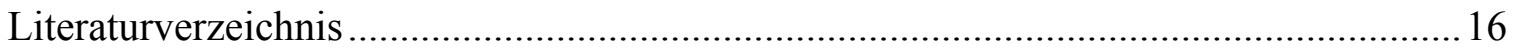

\section{Zur Entwicklung des Controlling als Fachgebiet innerhalb der Betriebswirtschaftslehre}

\subsection{Controlling als Phänomen in der unternehmerischen Pra- xis}

Controlling als Bezeichnung für einen - im Folgenden noch näher zu umreißenden Aufgabenbereich im Unternehmen bzw. die Position des Controllers (Controllership) ließ sich zunächst als Phänomen innerhalb der unternehmerischen Praxis beobachten. ${ }^{1}$ Ausgangspunkt dieser Entwicklung waren die USA, in denen spätestens seit der ersten Hälfte des 20. Jahrhunderts Controllerstellen für die Bearbeitung bestimmter Aufgaben inner-

Ausführlichere, inhaltlich im Wesentlichen übereinstimmende Darstellungen der Entwicklung des Controlling finden sich in vielen Standard-Lehrbüchern zum Controlling, so z.B. bei Hahn/Hungenberg (2001), S. 265-271, Horvàth (2001), S. 28-84, Küpper (2001), S. 1ff., oder Weber (1999), S. 1-29. 
halb des financial management eingerichtet wurden. Typische Controlleraufgaben umfassten nach dem Katalog des Controller's Institute of America (heute: Financial Executives Institute, FEI) vor allem die Durchführung der Planung, die Berichterstattung und Interpretation, die Bewertung und Beratung bei betriebswirtschaftlichen Fragestellungen sowie Revisions- und Kontrolltätigkeiten. ${ }^{2}$

In deutschen Unternehmen wurden Controllerstellen vermehrt erst seit den fünfziger Jahren des 20. Jahrhunderts eingerichtet, wobei die Tochtergesellschaften amerikanischer Unternehmen eine Vorreiterrolle spielten. Bereits Ende der siebziger Jahre fanden sich dann in fast allen größeren Unternehmen Controllerstellen. ${ }^{3}$ Jüngere Untersuchungen bestätigen die Gültigkeit dieses Bildes auch für den heutigen Zeitpunkt. ${ }^{4}$

Eine tiefergehende empirische Analyse zeigt, dass die den Controllern in deutschen Unternehmen zugeordneten funktionalen Tätigkeiten innerhalb der Unternehmensführung im Zeitverlauf einem erheblichen Wandel unterlagen. ${ }^{5}$ Während zunächst Aufgaben im Bereich Kostenrechnung und Kalkulation dominierten, trat deren Bedeutung kontinuierlich zurück. Im Gegenzug gewannen das Berichtswesen und die operative Planung an Relevanz, ebenso die Budgetierung und Budgetkontrolle sowie Abweichungsanalysen und Kostenüberwachungen. Die Mitwirkung in der strategischen Planung stellte insbesondere zu Beginn der achtziger Jahre eine wichtige Controlleraufgabe dar, hat bis heute jedoch insgesamt wieder an Stellenwert verloren.

Vergleicht man die aktuelle Situation mit den USA, so zeigt sich interessanterweise, dass dort im Gegensatz zu Deutschland der Begriff des Controlling als institutionale Abgrenzung bestimmter Aufgabengebiete kaum noch gebräuchlich ist. So spricht die Mehrzahl der Unternehmen nicht mehr von controller's department oder controllership, vielmehr werden die entsprechenden Funktionen durch das finance department oder das accounting department wahrgenommen. ${ }^{6}$ Plakativ sei an dieser Stelle Vaassen zitiert, der den Controller als ,generalist ... pulling the financial strings ${ }^{67}$ beschreibt.

2 Vgl. Financial Executives Institute (1962), S. 289.

3 Vgl. Horváth (2001), S. 59, unter Bezugnahme auf eine Untersuchung des Lehrstuhls aus dem Jahre 1978.

$4 \quad$ Vgl. z.B. Stoffel (1995), S. 132-141.

5 Vgl. hierzu die Analyse von Controlleraufgaben in deutschen Unternehmen auf der Basis von Stellenanzeigen von 1949-1994 bei Weber/Kosmider (1991), S. 28, sowie ergänzend Weber/Schäffer (1998), S. 229.

6 Vgl. Stoffel (1995), S. 140.

7 Vaassen (2002), S. 7. 


\subsection{Controlling in der betriebswirtschaftlich-theoretischen Diskussion}

In der deutschen Betriebswirtschaftslehre wird das Phänomen Controlling als Reflex auf dessen zunehmende praktische Relevanz vor allem seit den siebziger Jahren diskutiert. Als wichtiger inhaltlicher und methodischer Promotor der frühen Zeit kann Horváth angesehen werden, dessen Lehrbuch „Controlling“ erstmals 1979 erschien. Ab diesem Zeitpunkt werden an deutschen Hochschulen in wachsender Zahl auch ControllingLehrstühle eingerichtet, die entweder eigenständig sind oder oft auch angrenzende Lehrgebiete umfassen. Hierzu gehört insbesondere die interne Rechnungslegung, vielfach aber findet sich Controlling als Lehrgebiet auch in Verbindung mit der Produktionswirtschaft im weiteren Sinne oder der Wirtschaftsprüfung. ${ }^{8}$

Seit den achtziger Jahren prägten zunehmend Persönlichkeiten wie Küpper oder Weber die betriebswirtschaftliche Diskussion um das Controlling. ${ }^{9}$ Eine Zwischenstellung nehmen Autoren wie z.B. Hahn ein, die die betriebswirtschaftliche Diskussion des Controlling unter einem anderen Rubrum - hier z.B. Planung und Kontrolle - beeinflusst haben. ${ }^{10}$ Insgesamt ist dabei mit Horváth jedoch festzuhalten, dass ,nicht die einzelnen In-

8 Vgl. z.B. Handelshochschule Leipzig (Rechnungswesen und Controlling, Thomas Fischer), Hochschule St. Gallen (Rechnungslegung und Controlling, Reiner Fickert), Private Universität Witten-Herdecke (Controlling und Informationsmanagement, Friederike Wall), RWTH Aachen (Unternehmenstheorie, Umweltökonomie und industrielles Controlling, Harald Dyckhoff), TH Darmstadt (Rechnungswesen und Controlling, Hans-Jürgen Wurl), TU Dresden (Rechnungswesen und Controlling, Thomas Günther), Universität Augsburg (Controlling und Wirtschaftsprüfung, Adolf G. Coenenberg), Universität Bern (Accounting and Controlling, Klaus Dellmann), Universität Dortmund (Unternehmensrechnung und Controlling, Thomas Reichmann), Universität Frankfurt (Controlling und Auditing, Ralf Ewert), Universität Graz (Unternehmensführung und Controlling, Alfred Wagenhofer), Universität Gießen (Industrielles Management und Controlling, Dietger Hahn), Universität Magdeburg (Unternehmensrechnung und Controlling, Alfred Luhmer), Universität München (Produktionswirtschaft und Controlling, Hans-Ulrich Küpper), Universität Münster (Controlling, Dieter Berens; Wirtschaftsinformatik und Controlling, Lothar Grob), Universität Stuttgart (Controlling, Péter Horváth), Universität Trier (Wirtschaftsprüfung und Controlling, Dieter Rückle), Universität Zürich (Interne Unternehmensrechnung und Controlling, Dieter Pfaff), WHU Otto-Beisheim-Hochschule (zunächst Controlling und Rechnungswesen, später Controlling und Logistik, heute Controlling und Telekommunikation, Jürgen Weber).

9 Ein wichtiger Ausgangspunkt sind hier die zwölf Thesen zum „Verständnis und Selbstverständnis des Controlling“ von Küpper/Weber/Zünd, publiziert in der ZfB im Jahre 1990; vgl. hierzu auch die Antworten von Deyhle (1991) und Remmel (1991). Das Lehrbuch von Weber erschien erstmals 1988 und hat bis heute ebenso wie das Werk von Horváth die achte Auflage erreicht. Küpper publizierte bis zu Beginn der neunziger Jahre im Bereich Controlling vor allem Aufsätze (1987, 1988, 1990); sein Lehrbuch erschien in der ersten Auflage 1995.

10 Vgl. hierzu das inzwischen in der sechsten Auflage erschienene Lehrbuch von Hahn/Hungenberg (2001). 
strumente und die Einzelaufgaben [innerhalb des Controlling, d.Verf.] neu sind, sondern deren Verknüpfung ${ }^{\text {"11 }}$.

In den USA ist eine vergleichbare Diskussion innerhalb der Gebiete business administration bzw. management nicht zu beobachten. Zwar werden dort unter dem Stichwort management control bzw. controllership durchaus einzelne Lehrbücher publiziert. ${ }^{12}$ Die im

deutschen Kontext innerhalb des Controlling diskutierten Problemstellungen (Planung, Kontrolle, Rechnungswesen) werden aber schwerpunktmäßig in anderen Fachgebieten wie accounting, cost management oder strategic management behandelt. In der USamerikanischen Forschung ist der Begriff des Controlling bzw. der Controllership damit heute - ganz im Gegensatz zur ersten Hälfte des 20. Jahrhunderts - faktisch bedeutungslos. ${ }^{13}$ Bisher gibt es hierfür kaum Erklärungen; eine Begründung könnte allerdings darin liegen, dass der englischsprachige Ausdruck to control inhaltlich mit steuern bzw. lenken zu übersetzen ist und damit weniger auf die Controllertätigkeit als solche, sondern auf originäre Aktivitäten des Managements abzielt.

Fasst man die betriebswirtschaftliche Diskussion um das Controlling in Deutschland zusammen, so können vor allem zwei sich zeitlich überlagernde Schwerpunkte bezogen auf die Entwicklung einer Controllingtheorie beobachtet werden.

- Begriffsfindung und Konzeptualisierung des Controlling:

Insbesondere in der Zeit ab Ende der siebziger Jahre bis zu Beginn der neunziger Jahre ging es vor allem darum, das Controlling gegenüber anderen betriebswirtschaftlichen Bereichen inhaltlich abzugrenzen und als eigenständiges Fachgebiet zu begründen. Unter den andiskutierten Controllingkonzeptionen, die unter Bezeichnungen wie gewinnzielorientiert, informationsorientiert, führungsorientiert oder koordinationsorientiert geführt werden, ${ }^{14}$ konnte sich vor allem die letztgenannte etablieren. ${ }^{15}$ Dieser koordinationsorientierte Ansatz wird - wenn auch mit unterschiedlicher Akzentualisierung u.a. von Horváth und Küpper sowie bis Ende der neunziger Jahre auch von Weber - vertreten. Nach einer Phase des Stillstands in den letzten Jahren ist diese Diskussion ab 1999 wieder neu belebt worden, nachdem von Weber/Schäffer ein Controllingverständnis im Sinne einer Sicherstellung der Rationalität der Führung vorgeschlagen wurde. ${ }^{16}$

- Methodik der Problemlösung für Aufgaben innerhalb des Controlling:

11 Horváth (2001), S. 83.

12 Vgl. z.B. Willson/Roehl-Anderson/Bragg, Steven M. (1999), Anthony/Govindarajan (2000).

13 So auch Horváth (2001), S. 53.

14 Vgl. ausführlich Weber (1999), S. 19-30, Küpper (2001), S. 5-13.

15 Vgl. als Beleg auch die Übersicht bei Küpper (2001), S. 8f.

16 Vgl. Weber/Schäffer (1999) sowie die Beiträge von Wall (2000) oder Pietsch/Scherm (2001). 
In einem zweiten Diskussionsstrang, der vor allem seit Beginn der neunziger Jahre an Bedeutung gewonnen hat, geht es um die Frage, wie die inhaltlichen Aufgabenstellungen, die aus dem im ersten Schritt identifizierten Controllingbegriff resultieren, zu bewältigen sind. Insbesondere der Unternehmenspraxis nahestehende Auto

ren wie Horváth oder Hahn arbeiten hier vor allem in neoklassischer Perspektive mit Systembeschreibungen bzw. Systemanalysen und leiten daraus Gestaltungsvorschläge z.B. für die Unternehmensplanung, das Rechnungswesen oder das Berichtswesen her. ${ }^{17}$ Eher theoretisch ausgerichtete Autoren wie z.B. Ewert, Wagenhofer oder Pfaff arbeiten dagegen auf einer institutionenökonomisch fundierten Ebene mit Methoden der Informationsökonomie, ${ }^{18}$ d.h. untersuchen das Zusammenspiel ökonomischer Akteure unter Berücksichtigung von Interessenkonflikten und asymmetrischer Information und die daraus resultierenden Auswirkungen bzw. Gestaltungsvorschläge z.B. für Systeme der Budgetierung oder des Performance Measurement. ${ }^{19}$

Insgesamt lässt sich zum heutigen Zeitpunkt festhalten, dass die in der ersten Phase vorherrschende Herausbildung einzelner Controllingschulen im Sinne verschiedener Controllingkonzeptionen überwunden scheint. Die inhaltliche Abgrenzung, die nicht zuletzt wohl aus der Notwendigkeit herrührte, Controlling als eigenständiges Fachgebiet zu etablieren, tritt in ihrer Bedeutung zurück; in Theorie und Praxis stellt Controlling einen anerkannten Teilbereich der Betriebswirtschaftslehre dar. Dennoch kann derzeit noch nicht von einer allgemein akzeptierten integrativen Klammer gesprochen werden, wie sie in anderen betriebswirtschaftlichen Teilgebieten, wie z.B. dem Marketing, dem Produktionsmanagement oder dem Rechnungswesen, vorhanden ist.

Gleichzeitig diffundiert das Controlling heute zunehmend in andere betriebswirtschaftliche Fachgebiete hinein, was zum einen auf das Controlling als „Modetrend“20, zum anderen jedoch sicherlich auch auf die Universalität der angewendeten Problemlösungsmethoden zurückzuführen ist. Symptomatisch erscheint das jüngste Ergänzungsheft 2/2001 der Zeitschrift für Betriebswirtschaft. Hier werden unter dem Titel „Controlling-Theorie“ eine Reihe von Aufsätzen unter Bezeichnungen wie Erlöscontrolling, Beschaffungscontrolling, Produktionscontrolling, Kostencontrolling, Investitionscontrolling, Geschäftsbereichs-Controlling oder strategisches Controlling zusammengefasst, die in ihrem jeweili-

17 Vgl. hierzu plakativ die Lehrbücher von Horváth (2001) bzw. Hahn/Hungenberg (2001).

18 Der wohl grundlegende Beitrag in diesem Zusammenhang wurde von Ewert (1992) publiziert.

19 Diese Sichtweise findet sich insbesondere im Lehrbuch von Ewert/Wagenhofer, das unter dem Titel „Interne Unternehmensrechnung“ erstmals 1993 erschien und in 2000 bereits die 4. Auflage erreichte. Die Autoren beschreiben im Vorwort explizit den Inhalt ihres Lehrbuchs als ,die Darstellung der internen Unternehmensrechnung; der Schwerpunkt liegt auf Fragen des Kosten- und Erlösmanagements sowie Instrumenten des Controlling“ (Ewert/Wagenhofer (2001), S. VI). 
gen Gebiet - eben der Beschaffung, der Produktionstheorie, der Kosten- und Investitionsrechnung, der Organisation oder der strategischen Führung - richtungsweisende Beiträge darstellen. Inwieweit eine gemeinsame Klammer „Controlling“ konzeptionell darüber steht, bleibt jedoch offen; ein Titel „Betriebswirtschaftliche Theorie“ erschiene eher angebracht.

\subsection{Fazit: Quo vadis Controlling?}

Die wesentlichen Ergebnisse zum aktuellen Stand des Controlling als Fachgebiet innerhalb der deutschen Betriebswirtschaftslehre lassen sich wie folgt zusammenfassen:

- Controlling ist als betriebliche Funktion in deutschen Unternehmen weit verbreitet.

- Als betriebswirtschaftliche Disziplin hat sich Controlling in Deutschland etabliert.

- Trotz der intensiven konzeptionellen Diskussion insbesondere in den Anfangsjahren um die Eigenständigkeit des Fachgebiets fehlt jedoch eine allgemein akzeptierte integrative Klammer.

Damit stellt sich die Frage über die Zukunft des Controlling als betriebswirtschaftliches Fachgebiet. Das Fehlen eines vergleichbaren Forschungsstrangs in den USA könnte hier den Verdacht entstehen lassen, dass die angestrebte Eigenständigkeit des Controlling als betriebswirtschaftliche Teildisziplin gar nicht erreichbar ist. Im Folgenden wird angestrebt, diese spezifisch deutsche Controllingtheorie $\mathrm{zu}$ begründen und daraus Ansatzpunkte für die konzeptionelle Einordnung des Controlling innerhalb der Betriebswirtschaftslehre herzuleiten.

\section{Erkenntnisobjekt der Controllingforschung oder: Gibt es eine eigenständige Controllingtheorie?}

\subsection{Controlling als Reaktion auf den inhaltlichen Stillstand in der Kostenrechnungstheorie}

Versucht man in der deutschen betriebswirtschaftlichen Literatur zum Controllingbegriff eine gemeinsame Klammer zur Controllingpraxis in deutschen Unternehmen zu finden, die gleichzeitig auch eine Einordnung in die internationale Diskussion erlaubt, so kristallisiert sich hier die Rechnungslegung, verstanden in einer sehr weiten Sichtweise als "Instrument zur quantitativen wertmäßigen Abbildung von Wirtschaftsgütern zum Zwecke der Wertelenkung von Güterbeständen und -bewegungen“"21 als ein solcher Bezugspunkt

21 Chmielewicz (1982), S. 13. 
heraus. Einige - an dieser Stelle lediglich beispielhaft zu verstehende Belege - stützen diese Sichtweise:

- Hahn/Hungenberg definieren Controlling als ,informationelle Sicherung ergebnisorientierter Führung“ auf der Grundlage des „Zahlenwerk[s] des Rechnungs- und Finanzwesens ${ }^{\text {‘22 }}$,

- Horváth spricht von einer „ergebniszielorientierten Koordination“23 als Aufgabe des Controlling,

- Küpper beschreibt als zentrale übergreifende Koordinationsinstrumente typische Teile des Rechnungswesens, wie Budgetierungssysteme, Kennzahlensysteme oder Verrechungs- und Lenkpreissysteme, ${ }^{24}$

- Deyhle schließlich - hier als Vertreter der Unternehmenspraxis zu sehen - bezeichnet den Controller als ,wirtschaftliche[r]n Begleiter“, der als „Lieferant für Zahlen“ bzw. als „Planverkäufer“" agiert. ${ }^{25}$

Wenn aber die Rechnungslegung offensichtlich ein zentrales Element in unterschiedlichen Controllingkonzeptionen bzw. auch im Controllingverständnis der unternehmerischen Praxis darstellt, so drängt sich unmittelbar die Frage nach dem Bezug zwischen Rechnungslegung und Controlling auf. In dem vorliegenden Beitrag wird hierzu die These vertreten, dass aus entwicklungshistorischer Perspektive die Bedeutung des Controlling in den achtziger und neunziger Jahren insbesondere aus dem inhaltlichen Stillstand innerhalb der Kostenrechnungstheorie resultiert. ${ }^{26}$

Die Rechnungslegung in Deutschland ist traditionell dadurch gekennzeichnet, dass zwischen externer und interner Rechnungslegung nicht nur funktional, sondern auch institutional streng differenziert wird. Diese institutionale Differenzierung betrifft neben der Unternehmenspraxis auch die Wissenschaft. In den allermeisten Fällen sind die Fachvertreter eindeutig in einem der beiden Bereiche positioniert. Eine gleichgewichtige Zuord

nung, wie sie noch bei Schmalenbach erfolgte, dessen Hauptwerke „Kostenrechnung und Preispolitik“ ${ }^{\star 27}$ sowie „Grundlagen dynamischer Bilanzlehre ${ }^{\text {(28 }}$ sowohl die interne als auch

22 Hahn/Hungenberg (2001), S. 265 bzw. S. 272.

23 Horváth (2001), S. 150.

24 Vgl. Küpper (2001), S. 26.

25 Alle genannten Zitate entstammen Deyhle (1989), S. 19f.

26 Dieser Stillstand wird bereits von Weber (1993), S. 5, konstatiert, der damit den provokanten Beitrag von Eberle (1989) mit dem Titel „Kosten- und Leistungsrechnung: Relevance Lost?“ aufgreift.

27 Dieses Werk wurde von Schmalenbach erstmals in Form zweier Aufsätze in der zfbf im Jahre 1919 publiziert.

28 Auch dieses Werk Schmalenbachs erschien erstmals 1919. 
die externe Rechnungslegung richtungsweisend beeinflussten, ist heute kaum noch zu beobachten. ${ }^{29}$

Innerhalb der internen Rechnungslegung dominierte die Kostenrechnungstheorie. Hier etablierten sich ab den sechziger Jahren neben der in der Schmalenbach'schen Denkweise verhafteten traditionellen Vollkostenrechnung und Zuschlagskalkulation zwei weitere konkurrierende Schulen: Die von Kilger konzeptualisierte Flexible (Grenz-) Plankostenrechnung und Deckungsbeitragsrechnung, die später insbesondere auch durch Plaut in die Praxis umgesetzt wurde, sowie die von Riebel vertretene Relative Einzelkosten- und Deckungsbeitragsrechnung. Gerade in diesen beiden Schulen wurden bis in die siebziger Jahre hinein die wesentlichen Neuerungen bezogen auf die interne Rechnungslegung entwickelt. ${ }^{30}$

Es ist zu vermuten, dass nicht zuletzt die zunehmend emotional gefärbte Auseinandersetzung - um nicht zu sagen, der Konkurrenzkampf - zwischen den verschiedenen Kostenrechnungsschulen dazu führte, dass eine Weiterentwicklung des internen Rechnungswesens, d.h. eines Rechnungswesens, dessen wesentliche Aufgabe in der Unterstützung der Führungstätigkeiten des Managements besteht, im Rahmen der bestehenden Strukturen kaum noch möglich war. Offensichtlich wird dies beispielsweise in der Auseinandersetzung der einzelnen Kostenrechnungsschulen ${ }^{31}$, vertreten insbesondere durch Laßmann, Kilger und Riebel, auf der 1979er Arbeitstagung der Kommission Rechnungswesen im Verband der Hochschullehrer für Betriebswirtschaft e.V.

Allerdings waren sowohl die flexible (Grenz-)Plankostenrechnung als auch die Relative Einzelkostenrechnung spätestens zu Beginn der achtziger Jahre nicht mehr in der Lage, auf die veränderten Rahmenbedingungen der Unternehmensinnen- und -umwelt zu reagieren. ${ }^{32}$ Typische Problemfelder der Praxis waren hier ein starkes Wachstum von Umsatz, Produktions- und Absatzregionen sowie der Mitarbeiterzahlen in vielen Unternehmen. Gleichzeitig wuchs der Veränderungsgrad interner Prozesse und damit auch die zugrunde liegenden güterwirtschaftlichen Strukturen zunehmend schnell. Beide Entwicklungen stellten steigende Anforderungen an die interne Rechnungslegung. Es reichte nicht mehr aus, durch ein ausgefeiltes System an Kostenfunktionen im Sinne der Kil-

29 Interessanterweise hat sich diese Verbindung von interner und externer Rechnungslegung im Verband der Hochschullehrer für Betriebswirtschaft e.V. bis heute gehalten: Die Kommission Rechnungswesen behandelt sowohl die interne als auch die externe Rechnungslegung. Bestrebungen der Vergangenheit, die dadurch vergleichsweise große Kommission zu teilen, konnten sich regelmäßig nicht durchsetzen.

30 Daneben existierten noch weitere Schulen wie z.B. die Betriebsplankostenrechnung, deren dominierende Vertreter Laßmann und Wartmann darstellen, vgl. m.w.N. Bungenstock (1995), S. 219. Eine vergleichbare Bedeutung wie die Kilger- bzw. Riebel-Schule konnten sie jedoch nicht erlangen.

31 Die Referate- und Diskussionsbeiträge sind bei Chmielewicz (1983) wiedergegeben; ein plakativer Zusammenschnitt der Diskussion findet sich bei Bungenstock (1995), S. XI-XII.

32 Vgl. kritisch hierzu neben dem Beitrag von Weber (1993), hier insbesondere S. 4f., auch Weber/Weißenberger (1997), S. 277ff. 
ger'schen Schule den Produktionsbereich des Unternehmens zu einem gegebenen Zeitpunkt abzubilden. Ebenso sprengte die wachsende Komplexität und Dynamik der Entwicklungen jede Möglichkeit, eine Grunddatenbank im Sinne der Riebel'schen Einzelkostenrechnung abzubilden.

Da eine Weiterentwicklung der internen Rechnungslegung im Kontext der klassischen Kostenrechnungstheorie nicht möglich war, bot der in der Praxis zunehmend etablierte Controllingbegriff den Rahmen für eine Diskussion der internen Rechnungslegung, die eben nicht mehr im Kontext starrer Kostenrechnungsschulen geführt werden musste. Vielmehr gab ein noch völlig offenes Fachgebiet „Controlling“ die Möglichkeit, frei von tradierten Denkweisen die notwendige Unterstützung der Unternehmensführung, d.h. die Entscheidungen über die Allokation knapper Ressourcen, durch das (interne) Rechnungswesen weiterzuentwickeln.

Diese Weiterentwicklung bestand jedoch nicht nur in der Fokussierung auf neue Formen der Kosten- (und Erlös)rechnung, wie beispielsweise der Prozesskostenrechnung oder des Target Costing. Ein Verdienst insbesondere der koordinationsorientierten Controllingansätze, repräsentiert durch wichtige Vertreter wie Hahn, Horváth, Küpper oder Weber, lag vor allem darin, die Abbildung güterwirtschaftlicher Vorgänge durch das Planungs- und Kontrollsystem im Hinblick auf die gestiegene Komplexität und Dynamik der Unternehmensum- und -innenwelt anzupassen. Gleichzeitig wurden dabei wichtige Schnittstellen z.B. in den Bereichen Organisationsgestaltung oder Performance Measurement nicht vernachlässigt. Parallel dazu erlaubte die Weiterentwicklung der internen Rechnungslegung unter dem Rubrum Controlling auch die fallbezogene Loslösung von Wertgrößen, wenn diese nicht bzw. nur ungenau zu generieren sind. Dies betrifft z.B. Kennzahlensysteme, wie sie u.a. im Logistikbereich entwickelt wurden, aber auch die in jüngerer Zeit intensiv diskutierten Balanced Scorecard-Konzepte.

\subsection{Controlling als Lehre von der Messung güterwirtschaftli- cher Sachverhalte für betriebliche Entscheidungen}

Wird die These akzeptiert, dass in der deutschen Betriebswirtschaftslehre notwendige Weiterentwicklungen im Bereich des internen Rechnungswesens unter dem Überbegriff Controlling stattgefunden haben, stellt sich die Frage, inwieweit Controlling dann auch heute noch als eigenständiges Gebiet in Forschung und Lehre geführt werden sollte. Diese Frage ist um so bedeutsamer, da es in der US-amerikanischen Forschung - wie oben dargestellt - eine vergleichbare Controllingforschung nicht gibt.

Berücksichtigt man jedoch, dass unter dem Begriff interne Rechnungslegung bzw. Kostenrechnung im Wesentlichen auch heute noch die tradierten Kostenrechnungsschulen 
verstanden werden ${ }^{33}$, steht $\mathrm{zu}$ befürchten, dass eine Neuorientierung des Fachgebietes Controlling unter dem Oberbegriff (interne) Rechnungslegung letztlich wieder in ein inhaltliches Stillstandsszenario vergleichbar zur Situation zu Beginn der achtziger Jahre mündet. Soll deshalb das Controlling als eigenständiges betriebswirtschaftliches Teilgebiet beibehalten werden, ist es dann allerdings notwendig, dessen Erkenntnisobjekt nicht nur phänomenologisch, d.h. bezogen auf die Gesamtheit der Entwicklung des Fachs seit $1980 \mathrm{zu}$ präzisieren. Vielmehr sollte abstrahierend ein gemeinsamer Kern aus diesen Entwicklungen gezogen werden.

An dieser Stelle wird vorgeschlagen, als einen solchen Kern Controlling als Lehre von der Messung güterwirtschaftlicher Sachverhalte für betriebliche Entscheidungen zu definieren. In einer zunehmend komplexen und dynamischen Umwelt besteht die Aufgabe des Controllers bzw. die daraus resultierenden Fragestellungen für die Controllingtheorie dann darin, für die vom Management sachlich definierten Problemstellungen bzw. Lösungsalternativen, also z.B. die Annahme eines Auftrags, die Neueinführung eines Produkts, die Umstrukturierung der Unternehmensorganisation, die Delegation bestimmter Aufgaben oder der Einsatz eines Entlohnungssystems, Messgrößen zu definieren, mit deren Hilfe das Management dann eine im betriebswirtschaftlichen Sinne richtige Entscheidung treffen kann, d.h. die zur Verfügung stehenden Ressourcen im Sinne des ökonomischen Prinzips effizient alloziiert. ${ }^{34}$ Solche Messgrößen sind in vielen Fällen monetäre Größen, also z.B. bezogen auf das Jahresergebnis oder einen gewünschten Unternehmenswert (shareholder value). Denkbar sind jedoch auch nichtmonetäre Messgrößen, wie z.B. das Erreichen eines bestimmten Marktanteils oder der Erfüllungsgrad von Kennzahlen wie Kundenzufriedenheit oder Liefertreue.

Die skizzierte Sichtweise des Controlling als Lehre von der Messung güterwirtschaftlicher Sachverhalte für betriebliche Entscheidungen hat verschiedene Eigenschaften:

- Zunächst lässt sich im Rahmen einer solchen Sichtweise unmittelbar ein eigenständiger Aufgabenbereich des Controlling im Gesamtkontext der Führung identifizieren. In einem sehr einfach strukturierten Umfeld ist durchaus denkbar, dass sowohl güterwirtschaftliche als auch messtheoretische Fragestellungen durch das Management uno actu gelöst werden. Mit einer zunehmend komplexer und dynamischer werdenden Unternehmensinnen- und -umwelt erscheint eine Aufspaltung der Gesamtführungsaufgabe in beide Teilaufgaben und Delegation der nachgelagerten Messung güterwirtschaftlicher Sachverhalte an einen Controller sinnvoll. Da dem Controlling in dieser Definition zudem alle, und nicht nur die monetär

33 Selbst Ewert/Wagenhofer (2000) haben in ihrem inhaltlich sehr stark an der internationalen Accounting-Diskussion anknüpfenden Lehrbuch „Interne Unternehmensrechnung“ ein eigenes Kapitel zu Kostenrechnungssystemen eingefügt, in dem speziell dieses Schulendenken aufgegriffen wird.

34 In diesem Sinne wird dann eine Informationsbeziehung zwischen Management und Controller etabliert, die selbst wieder Gegenstand betriebswirtschaftlicher Untersuchungen ist, vgl. z.B. Weißenberger (1997). 
formulierten, Messaufgaben für Zwecke der Führungsunterstützung zugeordnet werden, ist das Controlling nicht deckungsgleich mit dem (internen) Rechnungswesen zu verstehen; vielmehr stellt das interne Rechnungswesen dann einen Teilbereich des Controlling dar.

- Mit Hilfe des hier definierten Controllingverständnisses existiert ein Ansatzpunkt, mit dem der oben kritisierten undifferenzierten Diffundierung des Controllingbegriffs als ,Modetrend' in die verschiedensten ökonomischen Sachverhalte hinein entgegengewirkt werden kann: Nur noch dann, wenn in einem Beitrag die Messung güterwirtschaftlicher Fragestellungen im Vordergrund steht, z.B. die Entwicklung eines Kennzahlensystems im Logistikbereich oder die Konzeptualisierung von Methoden zur Messung der Kundenzufriedenheit, kann von Controlling, hier als Logistik- bzw. Marketingcontrolling präzisiert, gesprochen werden.

- Das vorgeschlagene Controllingverständnis im Sinne einer Messung güterwirtschaftlicher Sachverhalte besitzt zudem vielfältige Anknüpfungspunkte an die bisherige Diskussion innerhalb der deutschen Controllingtheorie. So ergibt sich beispielsweise die von einer Vielzahl von Autoren in unterschiedlichen Facetten vertretene Koordinationssicht des Controlling auch als Konsequenz der - vielfach, aber nicht ausschließlich monetären - Messung güterwirtschaftlicher Sachverhalte: Durch den dabei erzeugten einheitlichen numéraire im Walrasianischen Sinne wird eine Koordination verschiedener Führungsteilfunktionen überhaupt erst ermöglicht. Die sachliche Ausgestaltung der Koordination, so beispielsweise die Auswahl und Implementierung einer bestimmten Organisationsstruktur, bleibt dabei allerdings dem Management vorbehalten. ${ }^{35}$ Dem Controlling obliegt hier die Aufgabe, auf Ineffizienzen, z.B. einer zentralistischen Organisationsstruktur in Verbindung mit einer dezentralen Planung hinzuweisen (Messung ex post) und die möglichen Alternativen im Sinne der Messaufgabe für das Management zu bewerten (Messung ex ante). Auch das von Weber/Schäffer (1999) vertretene Verständnis von Controlling als Sicherstellung der Rationalität von Führung widerspricht nicht dem hier vorgestellten Ansatz; allerdings wird durch die Fokussierung auf Fragen der Messung das Objekt der Rationalitätssicherung vergleichsweise enger gefasst.

- Weiterhin lässt sich der vorgeschlagene Controllingbegriff unmittelbar in das Praxisverständnis des Controlling einordnen, das ja nicht nur eine im traditionellen Sinne verstandene interne Rechnungslegung umfasst. Vielmehr beinhaltet dieser Controllingbegriff u.a. auch Fragen des Berichtswesens, d.h. der standardisierten Abbildung güterwirtschaftlicher Sachverhalte, der Budgetierung, d.h. der monetären Abbildung zugewiesener Ressourcen sowie der operativen und strategischen Planung, die ausgehend von einer güterwirtschaftlich verstandenen Sachzielplanung in eine Formalzielplanung zu überführen ist. Genau diese angesprochenen

35 Anders z.B. Weber (1999), S. $277 \mathrm{f}$. 
Bereiche stellen in Deutschland wie auch international zentrale Controlleraufgaben dar. ${ }^{36}$

- Schließlich kann ein Controllingverständnis, das auf die Messung güterwirtschaftlicher Sachverhalte abzielt, intuitiv in eine internationale Diskussion integriert werden, die den Begriff des Controlling als solches nicht verwendet. Kommunikationsprobleme, die daraus resultieren, dass vor einer Präsentation der eigentlichen Sachprobleme erst eine umfangreiche und zeitraubende Definition des Controllingverständnisses zu erfolgen hat, werden damit vermieden.

Zwar schließt sich mit dem letztgenannten Punkt wieder der Kreis zu der eingangs gestellten Frage, inwieweit dem Controlling ein eigenständiges Erkenntnisobjekt insbesondere im Vergleich zur internen Rechnungslegung zuzuordnen ist. Akzeptiert man jedoch die enge Begriffsbelegung der Kostenrechnung bzw. der internen Rechnungslegung in der deutschen Betriebswirtschaftslehre, erscheinen die in den letzten Jahren unter dem Rubrum Controlling angesammelten Erkenntnisse als ausreichend, um Controlling auch weiterhin als Teilgebiet der betriebswirtschaftlichen Forschung und Lehre zu halten. Provokativ könnte die Frage nach der Eigenständigkeit des Controlling auch umgekehrt dahingehend gestellt werden, ob heute die Kostenrechnung bzw. interne Rechnungslegung noch ein eigenständiges Teilgebiet der Betriebswirtschaftslehre darstellen oder ob sie letztlich nur noch als Teilaspekt einer umfassenderen Controllingtheorie zu verstehen sind.

\section{Konsequenzen des vorgeschlagenen Controllingverständ- nisses für Forschung und (Hochschul-)Lehre}

Zum Abschluss des vorliegenden Beitrags soll skizziert werden, welche Konsequenzen aus der im vorangegangenen Abschnitt vorgeschlagene Sichtweise des Controlling, nämlich als Lehre von der Messung güterwirtschaftlicher Sachverhalte für betriebliche Entscheidungen, sowohl für die Forschung als auch für die universitäre Ausbildung resultieren können.

\subsection{Forschung}

Innerhalb der Forschung sind - abstrahiert man einmal von der Begriffsfindung, die durch den oben vorgestellten Definitionsansatz im Folgenden als abgeschlossen verstanden sein soll - zwei Aufgabenfelder zu differenzieren: ${ }^{37}$ Dies ist zum einen die Wirtschaftstheorie, in der es darum geht, Kausalitäten zu identifizieren, zum anderen die

\footnotetext{
36 Vgl. Stoffel (1995), S. 155ff.

37 Vgl. Chmielewicz (1994), S. 8-42.
} 
Wirtschaftstechnologie, in deren Kontext aus kausalen Zusammenhängen bestimmte ZielMittel-Relationen hergeleitet werden.

Für die Controllingforschung bedeutet dies, dass zum einen kausale Zusammenhänge zu untersuchen sind, wie beispielsweise die Frage nach der inhaltlich sinnvollen Abbildung bzw. Messung eines gegebenen güterwirtschaftlichen Sachverhalts; ein Beispiel bestünde hier in der kritischen Diskussion bzw. Weiterentwicklung der Prozesskostenrechnung. ${ }^{38}$ Gleichzeitig könnte untersucht werden, wie die Erfolgsmessung dezentraler Entscheidungsträger deren Verhalten beeinflusst; diese Frage wird derzeit unter dem Oberbegriff Performance Measurement bzw. Führungskräfteentlohnung intensiv diskutiert. ${ }^{39}$ Gleichzeitig muss die Controllingforschung aber auch in der Lage sein, diese Erkenntnisse in praktisch verwertbare Ziel-Mittel-Aussagen zu übersetzen, d.h. beispielsweise Anhaltspunkte dafür liefern, unter welchen Bedingungen eine bestimmte Ausgestaltungsform der Erfolgsmessung im Unternehmen zielführend einsetzbar ist.

Um diese Forschungsziele zu erreichen, kann zwischen neo-klassischen, heute meist systemorientiert formulierten Forschungssträngen sowie neo-institutionalen und behavioristisch ausgerichteten Ansätzen differenziert werden.

Im ersten Fall einer neoklassisch/systemorientierten Forschungsrichtung wird von Informationsasymmetrien bzw. Interessenkonflikten zwischen Controller und Manager bzw. zwischen verschiedenen Stufen des Managements oder auch zwischen Eigentümern und Managern abstrahiert. Die Messung güterwirtschaftlicher Sachverhalte zielt auf eine Entscheidungsunterstützungsfunktion innerhalb der Unternehmensführung bei der Allokation knapper Ressourcen ab. Für die Controllingtheorie ist damit bedeutsam, wie Führungsprozesse im Einzelnen ablaufen, d.h. den Zyklus von Planung, Steuerung und Kontrolle zu verstehen, und an welchen Stellen die Übersetzung von der güterwirtschaftlichen in die typischerweise monetäre Ebene zu erfolgen hat.

Im zweiten Fall der neo-institutionalen Controllingforschung geht es nicht nur um die Identifikation bestimmter Entscheidungsprobleme als solche, sondern um die dahinterstehenden Interessenkonflikte zwischen den verschiedenen ökonomischen Akteuren. Aus Controllingsicht liegt der Fokus dann weniger auf der einfachen Übersetzung güterwirtschaftlicher Produktionsvorgänge, sondern vielmehr auf der Berücksichtigung des Einflusses einzelner Akteure auf diese Vorgänge: Es soll sichergestellt werden, dass durch die Art der Abbildung dieser Einfluss möglichst günstig im Sinne einer vorgegebenen Zielsetzung, z.B. der Maximierung des Unternehmenswerts aus Sicht der Eigenkapitalgeber, erfolgt.

38 Vgl. z.B. den Beitrag von Christensen/Demski (1997).

39 Vgl. zum Stand der Literatur ausführlich die Arbeit von Weißenberger (2002). 
Während bezogen auf die neoklassisch/systemorientierte bzw. die neo-institutionale Sichtweise bereits umfangreiche Forschungsergebnisse innerhalb der Controllingtheorie vorliegen, spielen behavioristisch ausgerichtete Untersuchungen bisher nur eine zurücktretende Rolle. ${ }^{40}$ Zielsetzung dieser Untersuchungen ist es, den Einfluss bestimmter psychologischer Konstrukte, wie Motivation, Einstellung, Wahrnehmung oder Erwartungsbildung sowohl auf die Abbildung güterwirtschaftlicher Vorgänge durch die Controller selbst als auch auf die Verarbeitung der vom Controlling generierten Wertgrößen durch das Management zu analysieren. Der Untersuchungsfokus ist damit vergleichbar zur neoinstitutionalen Sichtweise akteursbezogen. Während dort jedoch ex ante festgelegte Verhaltensweisen im Sinne des mikro-ökonomischen Kalküls einer zumindest nur beschränkt begrenzten Rationalität und Optimierung im Rahmen des Bernoulli-Prinzips zugrunde gelegt werden, stellt die behavioristische Sichtweise genau diese Verhaltensweise der Akteure in Frage.

Behavioristische Untersuchungsansätze gab es im Bereich Rechnungswesen vor allem in den sechziger und siebziger Jahren; ${ }^{41}$ allerdings wurden hier nur wenige verwertbare Ergebnisse erzielt. Anders jedoch z.B. im Marketing; hier stellen u.a. die behavioristischen Theorien zum Kaufverhalten einen bedeutenden Forschungszweig dar. Auch innerhalb der Finanzierungstheorie setzt sich unter dem Rubrum Behavioral Finance zunehmend eine verhaltensorientierte Interpretation bestimmter Kapitalmarktanomalien durch. ${ }^{42}$ Da in den letzten Jahren sowohl innerhalb der deskriptiven Entscheidungstheorie, der experimentellen Spieltheorie und nicht zuletzt auch innerhalb der Medizin bzw. der Psychologie wichtige Fortschritte erzielt wurden, ${ }^{43}$ erscheint es fruchtbringend, neben der notwendigen Weiterverfolgung des neoklassisch/systemorientierten bzw. des neo-institutionalen Ansatzes auch einen Forschungszweig Behavioral Controlling zu etablieren.

\section{2 (Hochschul-)Lehre}

Aufgabe der Lehre ist es zum einen, den Studenten die wissenschaftliche Auseinandersetzung mit Fragen der Controllingtheorie nahe zu bringen, zum anderen, sie auf die praktische Umsetzung dieses Wissens im Unternehmen vorzubereiten. Ein theoretisches Controllingverständnis sollte vor diesem Hintergrund zum einen die Controllingaufgaben vergleichsweise trennscharf identifizieren und zum anderen weitgehend auch die in der Praxis als solche wahrgenommenen Controlleraufgaben umfassen. Beides wird nach hier

40 Vgl. hier jünger u.a. die Untersuchung von Shields/Deng/Kato (2000).

41 Vgl. z.B. Kappler (1974).

42 Vgl. im Überblick Behavioral Finance Group (2000).

43 So wurden z.B. in der Medizin und Psychologie unter der Bezeichnung ,evidence-based best practice“ Verfahren entwickelt, mit deren Hilfe aus einer Vielzahl individuell nicht reproduzierbarer empirischer Studien eines bestimmten Phänomens dennoch verallgemeinerbare Aussagen abgeleitet werden können. 
verstandener Auffassung durch die vorgeschlagene Controllingdefinition als Lehre von der Messung güterwirtschaftlicher Sachverhalte für betriebliche Entscheidungen geleistet.

Die betriebswirtschaftliche Hochschullehre sollte sinnvollerweise insbesondere solche Forschungsrichtungen reflektieren, die sich weitgehend etabliert haben. Dazu gehört im Bereich der Controllingtheorie sowohl der neoklassisch/systemorientierte als auch der neo-institutionale Forschungszweig. Ersterem lassen sich beispielsweise Autoren wie Hahn oder Horváth zuordnen, der zweite Zweig wird u.a. von Ewert, Pfaff oder Wagenhofer vertreten. Flankierend sind allerdings auch ergänzende Themen der praktischen Controllertätigkeit wie z.B. Präsentationstechnik, Gesprächsführung, Konfliktbewältigung sowie die graphische Umsetzung von Zahlenmaterial als Element der Lehre zu etablieren.

Strukturell sollte die studentische Ausbildung zunächst einen unmittelbar praxisorientierten Einblick in Führungs- und Entscheidungsprozesse geben, um damit Ansatzpunkte für die wertmäßige Abbildung güterwirtschaftlicher Sachverhalte im Rahmen des Controlling aufzuzeigen. Eine detaillierte Kenntnis der einzelnen Führungsteilsysteme ${ }^{44}$, wie Planungs-, Kontroll-, Informations-, Personalführungs- oder Organisationssystem und der dort eingesetzten Instrumente ist dabei ebenso von Bedeutung wie eine intensive Auseinandersetzung mit Fragen der Rechnungslegung.

Daneben muss in der Lehre jedoch auch sichergestellt werden, dass die Studenten im Rahmen ihrer Ausbildung lernen, Informationsstrukturen und ihren Einfluss auf das Verhalten der betrachteten Akteure im Lichte gegebener Annahmen zu identifizieren. Dabei geht es insbesondere um Budgetierungsrechnungen, Erfolgsmessung zum Zweck der Anreizgestaltung, der Investitionsrechnung, Verrechnungspreisschemata oder Kostenallokationen. Gleichzeitig müssen die Studenten jedoch auch in der Lage sein, die häufig sehr abstrakt formulierten Ergebnisse in die unternehmerische Praxis zu übertragen.

Aufbauend auf einer betriebswirtschaftlichen Basisausbildung im Rahmen des Grundstudiums bzw. eines Bachelor-Studiengangs könnte das Lehrprogramm im Bereich Controlling für das Hauptstudium bzw. einen Master-Studiengang beispielhaft derart ausgestaltet sein, dass in einem ersten Schritt zunächst im Rahmen einer neoklassisch/systemorientierten Betrachtung die Führungsteilsysteme, insbesondere das Informationssystem, und die praktische Controllertätigkeit vorgestellt wird. Durch die zunehmende Verzahnung von interner und externer Rechnungslegung sollte die Ausbildung beide Gebiete umfassen und dabei im Sinne der Internationalisierung der Rechnungslegung neben einer Vermittlung nationaler Standards sowohl die International Accounting Standards (IAS, zukünftig IFRS) bzw. die US-GAAP beinhalten.

44 Weber (1999), S. 43, spricht in diesem Zusammenhang von Bausteinen, die für die Sicherstellung der Führungsrationalität erforderlich sind. 
Im zweiten Schritt werden dann einzelne Instrumente, wie z.B. die Budgetierung, herausgegriffen, und es werden mikroökonomische Implikationen dieser Instrumente im Lichte der neo-institutionalen Modellierung erarbeitet. Denkbar wäre hier z.B. der Sperrklinkeneffekt, der besagt, dass im mehrperiodigen Kontext erwartete zukünftige Anpassungen der Vorgaben bei hoher Leistung dazu führen, dass in den Anfangsperioden ein eher niedriges Leistungsniveau gewählt wird.

In einem dritten und letzten Schritt sind die einzelnen Instrumente zur Abbildung güterwirtschaftlicher Sachverhalte noch einmal zu vertiefen, um sowohl eine gewisse Anwendungsroutine sicherzustellen, als auch um die neo-institutionalen Erkenntnisse - soweit möglich - wieder in praktische Überlegungen umzusetzen. Behavioristische Aspekte können dabei - soweit im Zeitverlauf gesicherte Erkenntnisse erarbeitet werden - eine ergänzende Rolle spielen. Inwieweit sie in kommenden Jahren eine eigenständige Stellung im Rahmen des Lehrprogramms einnehmen werden, muss letztlich die Zukunft in diesem Forschungsfeld zeigen.

\section{Literaturverzeichnis}

Anthony, R.N./Govindarajan, V. (2000): Management Control System, 10. Aufl., New York et al.

Behavioral Finance Group (2000): Behavioral Finance - Idee und Überblick, in: Finanz Betrieb, 2. Jg., S. 311-318.

Bungenstock, C. (1995): Entscheidungsorientierte Kostenrechnungssysteme, Wiesbaden.

Chmielewicz, K. (1982): Betriebliches Rechnungswesen 1. Finanzrechnung und Bilanz, 3. Aufl., Opladen.

Chmielewicz, K. (Hrsg.) (1983): Entwicklungslinien der Kosten- und Erlösrechnung. Kommission Rechnungswesen im Verband der Hochschullehrer für Betriebswirtschaft e.V., Stuttgart.

Chmielewicz, K. (1994): Forschungskonzeptionen der Wirtschaftswissenschaft, 3. Aufl., Stuttgart.

Christensen, J./Demski, J.S. (1997): Product costing in the presence of endogenous subcost functions, in: RAS, 2. Jg., S. 65-88.

Deyhle, A. (1989): Management- \& Controlling Brevier. Band I, Manager \& Controller im Team, 5. Aufl., Gauting.

Deyhle, A. (1991): Kommentar der 12 Thesen im Beitrag Küpper/Weber/Zünd zum "Verständnis und Selbstverständnis des Controlling", in: ZfB Ergänzungsheft 3/1991, 61. Jg., S. 1-7. 
Eberle, P. (1989): Kosten- und Leistungsrechnung: Relevance Lost?, in: DBW, 49. Jg., S. 97-113.

Ewert, R. (1992): Controlling, Interessenkonflikte und asymmetrische Information, in: BFuP, 44. Jg., S. 277-303.

Ewert, R./Wagenhofer, A. (1993): Interne Unternehmensrechnung, Berlin et al.

Ewert, R./Wagenhofer, A. (2000): Interne Unternehmensrechnung, 4. Aufl., Berlin et al.

Financial Executive Institute (1962): Controllership and Treasurership Functions Defined by FEI, in: The Controller, 30. Jg., S. 289.

Hahn, D/Hungenberg, H. (2001): PuK-Controllingkonzepte, 6. Aufl., Wiesbaden.

Horváth, P. (1979): Controlling, München.

Horváth, P. (2001): Controlling, 8. Aufl., München.

Kappler, E. (1974): Motivierung durch Kostenwertvorgabe? Einige Überlegungen zu möglichen Auswirkungen der Kostenwertvorgabe in Organisationen. In: zfbf, 26. Jg., S. 335-353.

Kilger, W. (1961): Flexible Plankostenrechnung, Opladen.

Kilger, W. (1992): Flexible Plankostenrechnung und Deckungsbeitragsrechnung, 9. Aufl., Wiesbaden.

Küpper, H.-U. (1987): Konzeption des Controlling aus betriebswirtschaftlicher Sicht, in: Scheer, A.-W. (Hrsg.): Rechnungswesen und EDV, Heidelberg, S. 82-116.

Küpper, H.-U. (1988): Koordination und Interdependenz als Bausteine einer konzeptionellen und theoretischen Fundierung des Controlling, in: Lücke. W. (Hrsg.): Betriebswirtschaftliche Steuerungs- und Kontrollprobleme, S. 163-183.

Küpper, H.-U. (1990): Industrielles Controlling, in: Schweitzer, M. (Hrsg.): Industriebetriebslehre, München, S. 785-892.

Küpper, H.-U. (1995): Controlling, Stuttgart.

Küpper, H.-U. (2001): Controlling, 3. Aufl., Stuttgart.

Küpper, H.-U./Weber, J./Zünd, A. (1990): Zum Verständnis und Selbstverständnis des Controlling, in: ZfB, 60. Jg., S. 281-293.

Pietsch, G./Scherm, E. (2001): Controlling - Rationalitätssicherung versus Führungs- und Führungsunterstützungsfunktion, in: DU, 55. Jg., S. 81-84.

Riebel, P. (1994): Einzelkosten- und Deckungsbeitragsrechnung, 7. Aufl., Wiesbaden.

Remmel, M. (1991): Zum Verständnis und Selbstverständnis des Controlling, in: ZfB Ergänzungsheft 3/1991, 61. Jg., S. 9-15.

Schmalenbach, E. (1919a): Selbstkostenrechnung (1), in: zfhf, 13. Jg., S. 257-299.

Schmalenbach, E. (1919b): Selbstkostenrechnung (2), in: zfhf, 13. Jg., S. 321-356.

Schmalenbach, E. (1925): Grundlagen dynamischer Bilanzlehre, Leipzig.

Shields, M.D./Deng F.J./Kato, Y. (2000): The Design and Effects of Control Systems: Test of Direct- and Indirect-effect models, in: AOS, 25. Jg., S. 185-202.

Stoffel, K. (1995): Controllership im internationalen Vergleich, Wiesbaden. 
Vaassen, E.H.J. (2002): Accounting Information Systems. A Managerial Approach, New York.

Wall, F. (2000): Koordinationsfunktion des Controlling und Organisation - Überlegungen zur Eigenständigkeit eines koordinationsorientierten Controlling, in: krp, 44. Jg., S. 295-304.

Weber, J. (1988): Einführung in das Controlling, Stuttgart.

Weber, J. (1993): Kostenrechnung im System der Unternehmensführung, in: Weber, J. (Hrsg.): Zur Neuausrichtung der Kostenrechnung, Stuttgart, S. 1-78.

Weber, J. (1999): Einführung in das Controlling, 8. Aufl., Stuttgart.

Weber, J./Kosmider, A. (1991): Controlling - Entwicklung in der Bundesrepublik Deutschland im Spiegel von Stellenanzeigen, in: ZfB, 61. Jg., Ergänzungsheft 3/1991, S. 17-35.

Weber, J./Schäffer, U. (1998): Controlling-Entwicklung im Spiegel von Stellenanzeigen 1990-1994, in: krp, 42. Jg., S. 227-233.

Weber, J./Schäffer, U. (1999): Sicherstellung der Rationalität von Führung als Aufgabe des Controlling, in: DBW, 59. Jg., S. 731-746.

Weber, J./Weißenberger, B.E. (1997): "Relative Einzelkosten- und Deckungsbeitragsrechnung". A Critical Evaluation of Riebel's Approach, in: MAR, 8. Jg., S. 277298.

Weißenberger, B.E. (1997): Die Informationsbeziehung zwischen Management und Rechnungswesen, Wiesbaden.

Weißenberger, B.E. (2002): Anreizkompatible Erfolgsmessung im Kontext der Konzernsteuerung, Habilitationsschrift, Vallendar.

Willson, J.D./Roehl-Anderson, J./Bragg, S.M. (1999): Controllership: The Work of the Managerial Accountant, 6. Aufl., New York. 
- 19 - 